\title{
Preface to the special collection of Induced Earthquakes
}

\author{
HongFeng Yang ${ }^{1 *}$, XiaoWei Chen ${ }^{2}$, Rebecca Harrington ${ }^{3}$, and YaJing Liu ${ }^{4}$ \\ 'Earth System Science Programme, Faculty of Science, The Chinese University of Hong Kong, Shatin, NT, Hong Kong, China; \\ ${ }^{2}$ School of Geosciences, The University of Oklahoma, Norman, Oklahoma, USA; \\ 3Institute for Geology, Mineralogy \& Geophysics, Ruhr University Bochum, Bochum, Germany; \\ ${ }^{4}$ Department of Earth and Planetary Sciences, McGill University, Montréal, Québec, Canada
}

Citation: Yang, H. F., Chen, X. W., Harrington, R., and Liu, Y. J. (2021). Preface to the special collection of Induced Earthquakes. Earth Planet. Phys., 5(6), 483-484. http://doi.org/10.26464/epp2021057

It has been long recognized that a variety of anthropogenic activities may cause earthquakes (Ellsworth et al., 2013; Yang HF et al., 2017). In the recent decades, induced earthquakes have been found in many settings and become a growing concern, in particular for regions that are undergoing with resource development. For instance, damaging earthquakes in the shale gas fields of Sichuan Basin and Oklahoma have been suggested to be associated with hydraulic fracturing and wastewater disposal (Lei XL et al., 2020; Keranen et al., 2014), respectively. Understanding mechanisms of induced earthquakes is critical for reducing the associated risks, yet demands integrated efforts of seismic and geodetic monitoring, probing hydraulic properties of subsurface structure, as well as geomechanical modeling.

In this special collection, we present six papers with contents spanning from earthquake monitoring to geomechanical modeling. Wong et al. (2021) and Zhou PC et al. (2021) have applied machine learning techniques to earthquake detection from the data recorded by permanent and a temporary seismic network in the Weiyuan shale gas field, Sichuan Province, respectively. Their newly acquired catalogs show clear improvement compared with those network routine catalogs. Miao SY et al. (2021) developed a new method to locate earthquakes and applied it in an oilfield in Oman and the Changning shale gas field, Sichuan Province. Yang W et al. (2021) proposed a revised local magnitude formula with coefficients calibrated for earthquakes in the Southern Sichuan Basin. Barbour and Beeler (2021) conducted a systematic investigation on deriving poroelastic properties of the Arbukle group in Oklahoma, based on fluid-level response to teleseismic waves. Hemami et al. (2021) conducted 3D fully coupled poroelastic analysis of the Wilzetta fault system and its response to saltwater injection within the Arbuckle group. The following part includes details in each contributed paper.

For induced seismicity, a complete earthquake catalog is crucial in evaluating the spatial-temporal correlation with anthropogenic

Correspondence to: H. F. Yang, hyang@cuhk.edu.hk

Received 12 NOV 2021; Accepted 15 NOV 2021.

Accepted article online 15 NOV 2021.

(C)2021 by Earth and Planetary Physics. activities, however, routine monitoring network is often limited by the station coverage and processing power. Wong et al. (2021) have applied advanced machine learning technique on detecting phase arrivals on the permanent network in the Weiyuan shale gas field, Sichuan Province, China, and find clear improvement in the accuracy of identifying both $\mathrm{P}$ and $\mathrm{S}$ arrivals. Then they derive differential times from waveform correlation to build a high-resolution earthquake catalog of induced earthquakes in the Weiyuan Area. The improved resolution permits a detailed analysis of the induced earthquakes, including investigation of the spatial and temporal of seismicity surrounding the geological structures activated during a M5 sequence in September 2019. It highlights the need for enhanced detection in establishing the causal relationships between injection activity and fault activation.

Although the catalog can be improved by more advanced phase pickers, the catalog completeness is subject to the coverage of permanent stations, which is often sparse in regions with infrequent earthquakes before anthropogenic activities. To augment the permanent network, temporary arrays are usually deployed to improve the station coverage to enhance the monitoring power. Zhou PC et al. (2021) have utilized a dense one-year temporary seismic network covering the Weiyuan shale gas field, and have also applied machine learning technique to develop a more complete earthquake catalog. Their new catalog contains 60 times as many earthquakes as those in the Chinese Earthquake Network Center (CENC) catalog using sparsely distributed permanent stations. Their new catalog achieves a magnitude completeness of $M_{\llcorner} 0$. To better illuminate the spatial-temporal patterns of the seismicity and relationship with wells, they have further refined the earthquake locations. They first use detected explosions and earthquakes to refine the regional velocity model, and then improve the locations from the new velocity model. Their new location shows sequential migration patterns overlapped with horizontal well branches around several well pads. Their study demonstrates the applicability of machine-learning techniques in completing earthquake catalogs, which is crucial in understanding earthquake triggering processes.

Besides applying the new earthquake detector to find more accurate phase arrivals, locating microseismicity is crucial to monitor- 
ing anthropogenic activities and earthquake evolution. Miao SY et al. (2021) apply a new waveform-based location method that employs a hybrid multiplicative imaging condition to characteristic functions of seismic waveforms. Through comparison with other stacking methods and applying it to both real and synthetic data sets of seismicity related to oil and gas production, the authors demonstrate an improvement in location resolution. This method brings improvements particularly in cases where signal-to-noise ratios (SNRs) are poor, or first arrivals are emergent.

Another fundamental yet important question in building an earthquake catalog is to determine the magnitude of earthquakes. Earthquake local magnitude $\left(M_{\mathrm{L}}\right)$ accuracy is critical to seismic hazard and risk assessment. This is acutely true in environments prone to induced earthquakes where traffic light protocols rely heavily, if not entirely, on the reported local magnitudes. However, local magnitudes could be overestimated, up to 1 unit of magnitude, by the current CNSN national standard when stations of short epicentral distances $(<10 \mathrm{~km})$ are included, as is the case for induced seismicity monitoring by local dense arrays. The study by Yang W et al. (2021) addresses the urgent need by proposing a revised local magnitude formula with coefficients calibrated for earthquakes in the Southern Sichuan Basin. Tested with 7500 events recorded by a dense nodal array in 2019 near the Changning-Zhaotong shale gas field, the new formula significantly reduces the $M_{\mathrm{L}}$ overestimate at short distances (100 s of meters to $30 \mathrm{~km}$ ). Combined with a machine learning technique for phase picking and event detection, this study provides an enriched, reliable seismicity catalog for local seismic risk characterization. The protocols of $M_{\mathrm{L}}$ coefficient optimization can potentially be applied to other regions and/or future dense array deployments for $M_{\mathrm{L}}$ improvement.

Although seismic monitoring is crucial for investigating induced earthquakes, understanding mechanisms responsible of inducing earthquakes and evaluating risks of future induced earthquakes demand geomechanical models that consider the interaction between fluids and rocks. Hemami et al. (2021) apply a 3D fully coupled poroelastic model on the Wilzetta fault system and compute its response to saltwater injection in the subsurface layers, especially the Arbuckle group and the basement. By setting up 3D fault geometries, they compute stress perturbations on the fault system considering multiple scenarios based on assumptions that had to be made in hydraulic relationships between the geological layers and fault zone. Nevertheless, numerical results show that injection of large volumes of fluid into the Brbuckle group tends to bring the part of the Wilzetta faults closer to failure.

One critical element in geomechanical modelling is how to define a reasonable range of model parameters, particularly for those without direct measurements. With near-field GPS network, hydraulic parameters of subsurface layers can be reasonably inferred (Jiang GY et al., 2020). However, dense near-field geodetic measurements are not always available in regions with induced earthquakes. Barbour and Beeler (2021) conduct a systematic investigation on deriving poroelastic properties of the Arbuckle group in Oklahoma using teleseismic surface waves. By monitoring the fluid-level changes in a repurposed Arbuckle disposal well in Sage County, Oklahoma and comparing with teleseismic waves recorded at a co-located broadband seismometer, they find signals of fluid level variation that correspond to the $S$ wave and
Love wave, in addition to the Rayleigh wave. Using a borehole strainmeter, they are also able to calibrate the dynamic strain inferred from broadband seismogram, which is then used to derive poroelastic parameters within the Arbuckle group. Furthermore, the poroelastic response of the Arbuckle formation is both azimuthally variable and anisotropic, which appears related to tectonic stress and strain indicators such as the orientations of the maximum horizontal stress and faults/fractures. The results also demonstrate a viable approach to estimate hydraulic properties from teleseismic waves.

While here we present a suite of studies with recent advances in investigating induced earthquakes in different settings, mechanism of induced earthquake is not fully clear, nor is there consensus on induced earthquake hazard mitigation. Therefore, further research relevant to induced earthquakes is in urgent need, particularly in the global trend towards carbon neutrality during which induced earthquakes will be inevitable when developing unconventional and green energy resources.

\section{References}

Barbour, A. J., and Beeler, N. M. (2021). Teleseismic waves reveal anisotropic poroelastic response of wastewater disposal reservoir. Earth Planet. Phys., 5(6), 547-558. https://doi.org/10.26464/epp2021034

Ellsworth, W. L. (2013). Injection-induced earthquakes. Science, 341(6142), 1225942. https://doi.org/10.1126/science.1225942

Hemami, B., Masouleh, S. F., and Ghassemi, A. (2021). 3D geomechanical modeling of the response of the Wilzetta Fault to saltwater disposal. Earth Planet. Phys., 5(6), 559-580. https://doi.org/10.26464/epp2021054

Jiang, G. Y., Qiao, X. J., Wang, X. Q., Lu, R. Q., Liu, L., Yang, H. F., Su, Y. D., Song, L. L., Wang, B. S., and Wong, T. F. (2020). GPS observed horizontal ground extension at the Hutubi (China) underground gas storage facility and its application to geomechanical modeling for induced seismicity. Earth Planet. Sci. Lett., 530, 115943. https://doi.org/10.1016/j.epsl.2019.115943

Keranen, K. M., Weingarten M., Abers G. A., Bekins B. A., and Ge S. (2014). Sharp increase in central Oklahoma seismicity since 2008 induced by massive wastewater injection. Science, 345(6195), 448-451. https://doi.org/10.1126/science.1255802

Lei, X. L., Su, J. R., and Wang, Z. W. (2020). Growing seismicity in the Sichuan Basin and its association with industrial activities. Sci. China Earth Sci., 63(11), 1633-1660. https://doi.org/10.1007/s11430-020-9646-X

Miao, S. Y., Zhang, H. J., Tan, Y. Y., and Lin, Y. (2021). Development of a new high resolution waveform migration location method and its applications to induced seismicity. Earth Planet. Phys., 5(6), 520-531. https://doi.org/10.26464/epp2021056

Wong, W. C. J., Zi, J. P., Yang, H. F., and Su, J. R. (2021). Spatial-temporal evolution of injection-induced earthquakes in the Weiyuan Area determined by machine-learning phase picker and waveform crosscorrelation. Earth Planet. Phys., 5(6), 485-500. https://doi.org/10.26464/epp2021055

Yang, H. F., Liu, Y. J., Wei, M., Zhuang, J. C., and Zhou, S. Y. (2017). Induced earthquakes in the development of unconventional energy resources. Sci. China Earth Sci., 60(9), 1632-1644. https://doi.org/10.1007/s11430-0179063-0

Yang, W., Chen, G. Y., Meng, L. Y., Zang, Y., Zhang, H. J. and Li, J. L. (2021). Determination of the local magnitudes of small earthquakes using a dense seismic array in the Changning-Zhaotong Shale Gas Field, Southern Sichuan Basin. Earth Planet. Phys., 5(6), 532-546. https://doi.org/10.26464/epp2021026

Zhou, P. C., Ellsworth, W. L., Yang, H. F., Tan, Y. J., Beroza, G. C., Sheng, M. H., and Chu, R. S. (2021). Machine-learning-facilitated earthquake and anthropogenic source detections near the Weiyuan Shale Gas Blocks, Sichuan, China. Earth Planet. Phys., 5(6), 501-519. https://doi.org/10.26464/epp2021053 